\title{
Sintonizador fuera de línea de un controlador PID discreto usando un algoritmo genético multiobjetivo
}

\author{
David Bedolla-Martínez ${ }^{1}$, Esther Lugo González², \\ Felipe Trujillo-Romero ${ }^{1}$, F. Hugo Ramírez Leyva ${ }^{3}$ \\ ${ }^{1}$ División de Estudios de Posgrado, Universidad Tecnológica de la Mixteca, \\ Huajuapan de León, Oaxaca, \\ México \\ ${ }^{2}$ CONACYT-Universidad Tecnológica de la Mixteca, Instituto de Electrónica y \\ Mecatrónica, Universidad Tecnológica de la Mixteca, \\ Huajuapan de León, Oaxaca, \\ México \\ ${ }^{3}$ Instituto de Electrónica y Mecatrónica, Universidad Tecnológica de la \\ Mixteca, Huajuapan de León, Oaxaca, \\ México \\ davidbedollamartinez@outlook.es, \{elugog, ftrujillo, hugo\}@mixteco.utm.mx
}

Resumen. Este trabajo presenta los resultados obtenidos, al usar un algoritmo genético multiobjetivo, para la sintonización de las ganancias de un controlador PID para el control de posición. Para evaluar este enfoque, la planta a controlar es un Robot Manipulador de 2 Grados de Libertad (GDL), que cuenta con las siguientes características: El modelo dinámico es No-Lineal, es un sistema de múltiples entradas y múltiples salidas (MIMO) y se tiene un acoplamiento en las dinámicas de cada articulación. Como resultado se obtuvo un conjunto de ganancias que estabilizan al sistema con un sobreimpulso y tiempo de establecimiento relativamente bajos.

Palabras clave: PID, algoritmo genético multiobjetivo, robot manipulador, sintonizador fuera de línea.

\section{Offline tuner of a discrete PID controller using a multi-objective genetic algorithm}

\begin{abstract}
This paper presents the results obtained when using a multi-objective genetic algorithm for tuning the gains of a PID controller for position control. To evaluate this approach, the plant to control is a robot manipulator with 2 degrees of freedom (DOF), which has the following characteristics: The dynamic model is not linear, it is a system of multiple input and multiple output (MIMO) and it has a coupling on the dynamics of each joint. As a result a set of gains that stabilize the system with an overshoot and settling time relatively low was obtained.
\end{abstract}


Keywords: PID, multi-objective genetic algorithm, robot manipulator, offline tuner.

\section{Introducción}

Sintonizar controladores de tipo Proporcional + Integral + Derivativo (PID), no es trivial, y a pesar de que existen diversos estudios para sintonizar estos controladores, parece no haberse resuelto el problema para tener una técnica que pueda ser implementada en los diversos sistemas, e.g. Los sintonizadores clásicos están limitados a sistemas de una entrada y una salida (SISO), de naturaleza lineal y que son estables en lazo abierto o los métodos inteligentes como las redes neuronales están limitados a los recursos computacionales [1]. El controlador PID no es el más adecuado para ciertos sistemas no lineales. Sin embargo, es el controlador preferido por la industria por la facilidad de implementarlos [2].

Para un controlador no es suficiente con mantener la respuesta deseada, también se pide que éste converja en un tiempo finito, otra manera de ver este acontecimiento, es llevar el error a cero en un lapso muy corto de tiempo sin tener grandes sobreimpulsos en la respuesta.

Estos requerimientos se modifican con un controlador PID y se varían a partir de sus ganancias, i.e., a partir de una configuración de ganancias, se tienen diferentes tiempos de establecimiento, sobreimpulso máximo de la planta, etc. Por tanto el objetivo es la búsqueda de ganancias que produzcan los requerimientos de diseño de tiempo y sobreimpulso mínimo.

Aunque los controladores PID son ampliamente usados en los procesos industriales, su efectividad es frecuentemente limitada debido a una sintonización pobre. La sintonización manual de las ganancias de un controlador es una tarea que consume demasiado tiempo [3]. Algunas investigaciones sobre la sintonización de controladores PID para sistemas MIMO se describen a continuación:

W.D. Chang [4] propone una modificación de la fórmula de recombinación de un algoritmo genético y este método se usa para determinar las ganancias de los controladores PID en proceso multivariables, i.e. sistemas MIMO. Donde se asegura que el algoritmo genético es uno de los métodos convincentes de búsquedas óptimas para la solución de problemas de control.

En [5] se presenta una comparación del desempeño de los algoritmos evolutivos para la sintonización de los controladores PIs y PIDs multivariables, tales algoritmos son: algoritmos genéticos de código real (RGA), optimización por cúmulo de partículas modificado (MPSO), la adaptación de la matriz de covarianza con estrategia de evolución (CMAES) y evolución diferencial (DE). A través de las simulaciones realizadas revelan que los cuatro algoritmos considerados son adecuados para la sintonización del controlador PID en modo fuera de línea. Sin embargo solo los algoritmos CMAES y MPSO son adecuados para la sintonización en línea del controlador PID.

En [6] se implementó un controlador PID para manipular la velocidad de un motor de $\mathrm{CD}$, donde el controlador fue sintonizado con un algoritmo genético e implementado en una FPGA. Sung-Kwun en [7] propone controladores difusos para estabilizar el sistema Viga-Bola, se compara con controladores PDs. La sintonización de los 
parámetros de estos controladores se realizó mediante un algoritmo genético basado en la competencia leal jerárquica (HFCGA, por sus siglas en inglés, hierarchical fair competition-based genetic algorithm). En [8] se sintoniza un controlador PID mediante un algoritmo genético multiobjetivo para un robot manipulador.

La problemática es la sintonización fuera de línea de 2 controladores PID para un robot de 2 GDL, el cual es un sistema no lineal tipo MIMO. Esta sintonización se realizará a partir de proponer las ganancias de los controladores, observando la respuesta del sistema con los controladores, evaluando el sobreimpulso máximo y tiempo de establecimiento repitiendo el proceso. Para automatizar este proceso se hará uso de un algoritmo genético multiobjetivo debido a que se requiere buscar el mínimo global de 4 variables, que son dos sobre impulsos máximos y dos tiempos de establecimiento.

\section{Sintonización fuera de línea}

\section{a. Robot Manipulador}

En la Fig. 1 se observa un manipulador Robótico, el cual consta de eslabones y articulaciones. Los eslabones sirven de soporte a las articulaciones que es en donde se genera el movimiento. La posición final del efector del robot está en función de la posición angular que adquiere cada articulación (coordenadas articulares). La posición final del robot $\mathbf{X}$ es un vector con tres componentes que se muestran en la ecuación (1):

$$
\boldsymbol{X}^{T}=\left[\begin{array}{lll}
x_{1} & x_{2} & x_{3}
\end{array}\right]^{T} .
$$

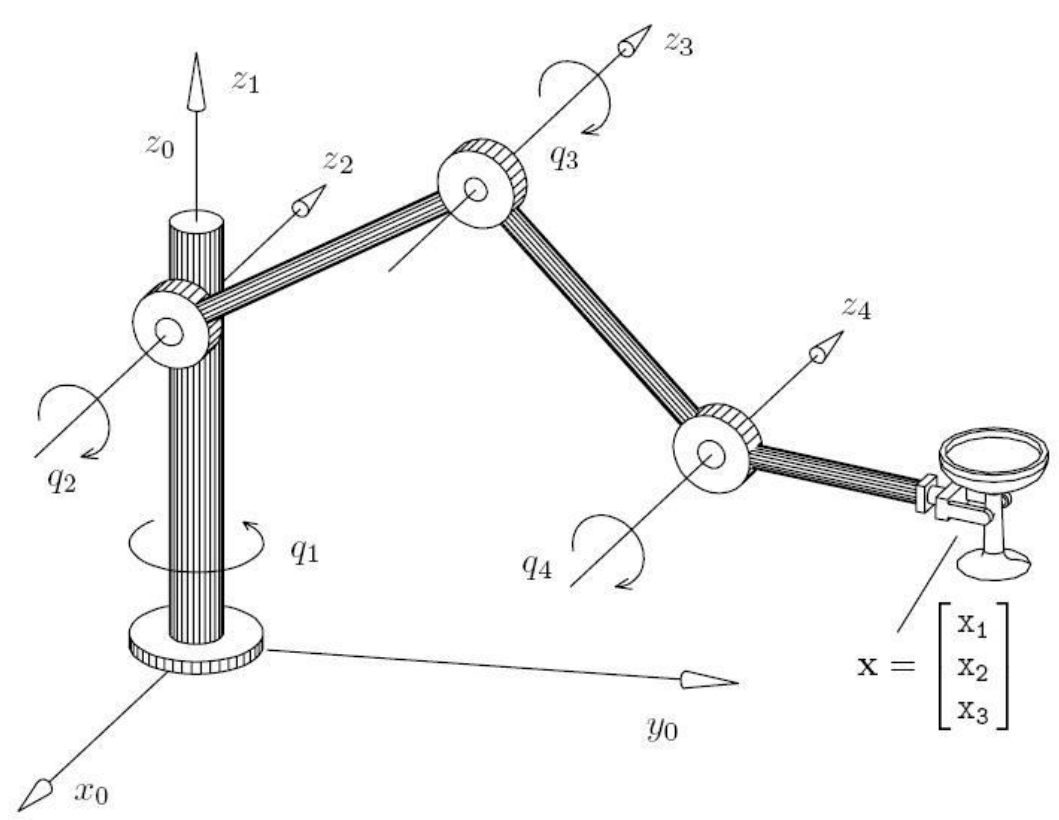

Fig. 1. Robot manipulador [9]. 
En forma general un robot se compone de $\mathrm{n}$ articulaciones, por lo cual el vector de posición $\boldsymbol{q}$ está dado por la ecuación (2), donde cada término corresponde a la coordenada de cada articulación:

$$
\boldsymbol{q}^{T}=\left[\begin{array}{llll}
q_{1} & q_{2} & \cdots & q_{n}
\end{array}\right]^{T} .
$$

$\mathrm{Al}$ generarse el movimiento del robot se tiene $\mathrm{n}$ velocidades que corresponden a la derivada de la posición y se muestran en la ecuación (3), donde $\dot{\boldsymbol{q}}$ corresponde a dicho parámetro:

$$
\dot{\boldsymbol{q}}^{T}=\left[\begin{array}{llll}
\dot{q}_{1} & \dot{q}_{2} & \ldots & \dot{q}_{n}
\end{array}\right]^{T} .
$$

Normalmente en cada articulación se tiene un motor que genera el par mecánico para mover la estructura mecánica. Para simplificar al sistema no se toma en cuenta la dinámica de éste y únicamente se usa como entrada el vector de par $\boldsymbol{\tau}$ de cada articulación, que está dado en la ecuación (4):

$$
\boldsymbol{\tau}^{T}=\left[\begin{array}{llll}
\tau_{1} & \tau_{2} & \cdots & \tau_{n}
\end{array}\right]^{T} .
$$

La posición $\boldsymbol{q}, \dot{\boldsymbol{q}}$ velocidad y $\boldsymbol{\tau}$ el par en cada articulación forma un vector. Mientras $\boldsymbol{X}$ es la posición del efector final.

Con base en la cinemática se determina el espacio de trabajo utilizando los parámetros Denavit-Hartenberg. En el modelado dinámico se obtienen las ecuaciones diferenciales que gobiernan el funcionamiento del sistema.

El modelado dinámico se obtiene a partir de las ecuaciones de Euler-Lagrange y el procedimiento para obtenerlo es el siguiente:

1. Obtener la cinemática directa

$$
\boldsymbol{X}=f\left(q_{1}, q_{2}, \ldots q_{n}\right)
$$

2. Modelo de energía

- Calculo de la energía cinética $K$

- Calculo de la energía potencial $U$

3. Cálculo del Lagrangiano

$$
L=K-U \text {. }
$$

4. Desarrollo de las ecuaciones de Euler-Lagrange

$$
\frac{d}{d t}\left(\frac{\partial L(\boldsymbol{q}, \dot{\boldsymbol{q}})}{\partial \dot{\boldsymbol{q}}}\right)-\frac{\partial L(\boldsymbol{q}, \dot{\boldsymbol{q}})}{\partial \boldsymbol{q}}=\boldsymbol{\tau} .
$$

La ecuación (7) representa a todas las ecuaciones diferenciales del sistema. Si el robot tiene 2 articulaciones esto implica que se tienen 2 ecuaciones diferenciales.

Si se utilizan directamente las ecuaciones obtenidas con Euler-Lagrange resultan ecuaciones diferenciales acopladas con complicada manipulación. Un enfoque 
alternativo es usar una representación general del sistema como se muestra en la ecuación (8) la cual permite desacoplar directamente las ecuaciones diferenciales [10].

Un robot Manipulador de 2 GDL se muestra en la Fig. 2. Está compuesto de 2 articulaciones, 2 eslabones y dos entradas de control. Los parámetros del sistema son: $m_{1}$ la masa del eslabón $1, l_{1}$ la longitud del eslabón $1, I_{1}$ la inercia del eslabón $1, l_{c 1}$ el centro de masa del eslabón $1, q_{1}$ la posición articular del eslabón $1, m_{2}$ la masa del eslabón $2, l_{2}$ la longitud del eslabón $2, I_{2}$ la inercia del eslabón $2, l_{c 2}$ el centro de masa del eslabón 2, $q_{2}$ la posición articular del eslabón 2.

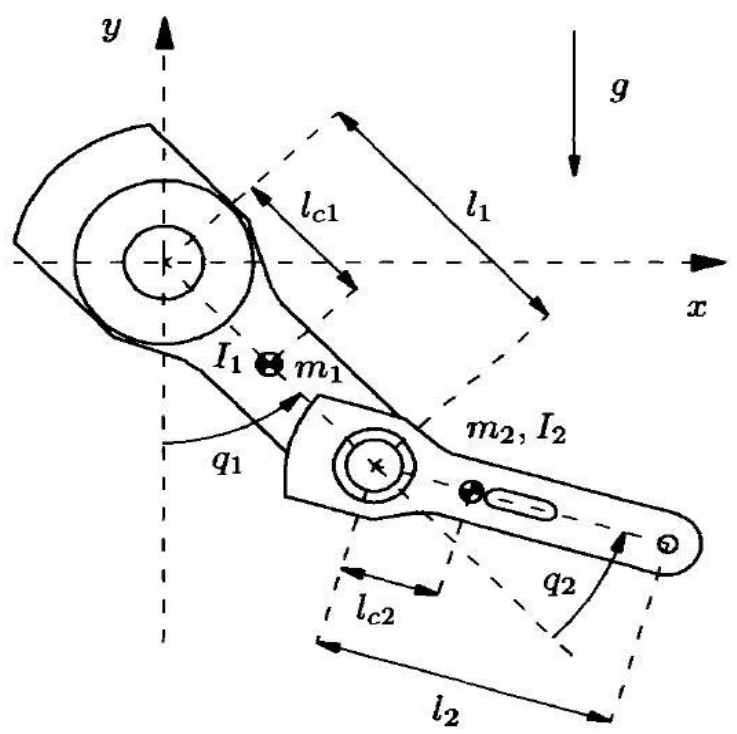

Fig. 2. Robot manipulador de 2 GDL.

El modelo dinámico se obtuvo de [11] y se muestra en la ecuación (8). Sus términos son: La matriz de Inercia $\boldsymbol{M}(\boldsymbol{q})$ y matriz de Coriolis $\boldsymbol{C}(\boldsymbol{q}, \dot{\boldsymbol{q}})$ son de $2 \mathrm{x} 2$, el vector de par gravitacional $\boldsymbol{g}(\boldsymbol{q})$, fricción $\boldsymbol{f}_{\boldsymbol{f}}(\dot{\boldsymbol{q}})$ son vectores de $2 \mathrm{x} 1$.

$$
\begin{gathered}
\boldsymbol{M}(\boldsymbol{q}) \ddot{\boldsymbol{q}}+\boldsymbol{C}(\boldsymbol{q}, \dot{\boldsymbol{q}}) \dot{\boldsymbol{q}}+\boldsymbol{g}(\boldsymbol{q})+\boldsymbol{f}_{\boldsymbol{f}}(\dot{\boldsymbol{q}})=\boldsymbol{\tau}, \\
\mathbf{M}(\boldsymbol{q})=\left[\begin{array}{ll}
M_{11} & M_{12} \\
M_{21} & M_{22}
\end{array}\right], \\
\boldsymbol{C}(\boldsymbol{q})=\left[\begin{array}{ll}
C_{11} & C_{12} \\
C_{21} & C_{22}
\end{array}\right], \\
\boldsymbol{g}(\boldsymbol{q})=\left[\begin{array}{l}
g_{1} \\
g_{2}
\end{array}\right], \\
\boldsymbol{f}_{\boldsymbol{f}}(\dot{\boldsymbol{q}})=\left[\begin{array}{l}
f_{f 1} \\
f_{f 2}
\end{array}\right] .
\end{gathered}
$$


Los parámetros del modelo son función de las coordenadas articulares, velocidades y posiciones articulares y los parámetros físicos del sistema están en las ecuaciones de la (9) a la (20), los cuales son función de los vectores de posición y velocidad articular. Donde la función $\operatorname{sat}\left(\tau, f_{e}\right)$ que depende del par de entrada y del coeficiente de fricción estática, representa el signo y magnitud del coeficiente de fricción estática.

Los parámetros que se muestran en la Tabla 1 pertenecen al robot manipulador de 2 GDL:

$$
\begin{aligned}
& M_{11}=m_{1} l_{c 1}^{2}+m_{2}\left[l_{1}^{2}+l_{c 2}^{2}+2 l_{1} l_{c 2} \cos \left(q_{2}\right)\right]+I_{1}+I_{2}, \\
& M_{12}=m_{2}\left[l_{c 2}^{2}+l_{1} l_{c 2} \cos \left(q_{2}\right)\right]+I_{2}, \\
& M_{21}=m_{2}\left[l_{c 2}^{2}+l_{1} l_{c 2} \cos \left(q_{2}\right)\right]+I_{2} \text {, } \\
& M_{22}=m_{2} l_{c 2}^{2}+I_{2}, \\
& \mathrm{C}_{11}=-m_{2} l_{1} l_{c 2} \operatorname{sen}\left(q_{2}\right) \dot{q}_{2}, \\
& \mathrm{C}_{12}=-m_{2} l_{1} l_{c 2} \operatorname{sen}\left(q_{2}\right)\left[\dot{q}_{1}+\dot{q}_{2}\right] \text {, } \\
& \mathrm{C}_{22}=0 \text {, } \\
& \mathrm{g}_{1}=\left[m_{1} l_{c 1}+m_{2} l_{1}\right] g \operatorname{sen}\left(q_{1}\right)+m_{2} l_{c 2} g \operatorname{sen}\left(q_{1}+q_{2}\right) \text {, } \\
& \mathrm{g}_{2}=m_{2} l_{c 2} g \operatorname{sen}\left(q_{1}+q_{2}\right), \\
& f_{f 1}=b_{1} \dot{q}_{1}+f_{c 1} \operatorname{signo}\left(\dot{q}_{1}\right)+\left[1-\left|\operatorname{signo}\left(\dot{q}_{1}\right)\right|\right] \operatorname{sat}\left(\tau_{1} ; f_{e 1}\right) \text {, } \\
& f_{f 2}=b_{2} \dot{q}_{2}+f_{c 2} \operatorname{signo}\left(\dot{q}_{2}\right)+\left[1-\left|\operatorname{signo}\left(\dot{q}_{2}\right)\right|\right] \operatorname{sat}\left(\tau_{2} ; f_{e 2}\right) \text {, } \\
& \operatorname{sat}\left(\tau, f_{e}\right)=\left\{\begin{array}{lr}
f_{e} & \text { si } \tau>f_{e} \\
\tau & \text { si }-f_{e} \leq \tau \leq f_{e} \\
-f_{e} & \text { si } \tau<-f_{e}
\end{array} .\right.
\end{aligned}
$$

Tabla 1. Parámetros del robot de 2 GDL.

\begin{tabular}{cc}
\hline Parámetro & Valor \\
\hline$l_{1}$ & $0.45 \mathrm{~m}$ \\
$l_{c 1}$ & $0.091 \mathrm{~m}$ \\
$l_{2}$ & $0.45 \mathrm{~m}$ \\
$l_{c 2}$ & $0.048 \mathrm{~m}$ \\
$m_{1}$ & $23.902 \mathrm{~kg}$ \\
$m_{2}$ & $3.88 \mathrm{~kg}$ \\
$I_{1}$ & $1.266 \mathrm{Nm} \mathrm{seg} / \mathrm{rad}$ \\
$I_{2}$ & $0.093 \mathrm{Nm} \mathrm{seg} / \mathrm{rad}$ \\
$b_{1}$ & $2.288 \mathrm{Nm} \mathrm{seg} / \mathrm{rad}$ \\
$b_{2}$ & $0.175 \mathrm{Nm} \mathrm{seg} / \mathrm{rad}$ \\
$\tau_{1}$ & $150 \mathrm{Nm}$ \\
$\tau_{2}$ & $15 \mathrm{Nm}$ \\
$g$ & $9.81 \mathrm{~m} / \mathrm{s}^{2}$ \\
\hline
\end{tabular}




\section{b. Algoritmo Genético Multiobjetivo}

Para sintonizar las ganancias de dos controladores PID se usó un algoritmo genético multiobjetivo, el cual busca disminuir el sobreimpulso máximo y el tiempo de establecimiento con un criterio del $2 \%$ de las 2 articulaciones del robot manipulador.

El algoritmo genético multiobjetivo se codificó usando toolbox de Matlab ®. Algunos criterios para la implementación del algoritmo genético son:

- El tipo de población es double

- El tamaño de la población es de 10,

- El tipo de selección es con torneos con un tamaño de 2,

- La probabilidad de cruce es de 0.8

- La probabilidad de mutación es de 0.001,

- El número de generaciones es de 100 ,

- El rango de búsqueda para la ganancia proporcional es de [0 300],

- El rango para la ganancia integral es de [0 600],

- El rango para la ganancia derivativa es de [0 50].

Las características anteriores dieron un tiempo de simulación de 2 minutos. La primera vez que se ejecutó el algoritmo genético tomó cerca de una hora debido a que las características eran diferentes como se muestra a continuación:

- El tamaño de la población era de 100 ,

- El número de generaciones era de 600,

- El rango de búsqueda para la ganancia proporcional era de [0 600],

- El rango para la ganancia integral era de [0 600],

- El rango para la ganancia derivativa era de [0 600].

Esto permitió reducir el rango de búsqueda de ganancias, el tamaño de la población y el número de generaciones con el fin de reducir el tiempo de simulación.

\section{c. Metodología}

Para cada individuo se proponen 6 ganancias 3 para cada controlador PID, se simula la planta con los controladores en lazo cerrado, posteriormente se evalúa el sobreimpulso máximo y el tiempo de establecimiento. En la Fig. 3 se muestra el diagrama del algoritmo genético.

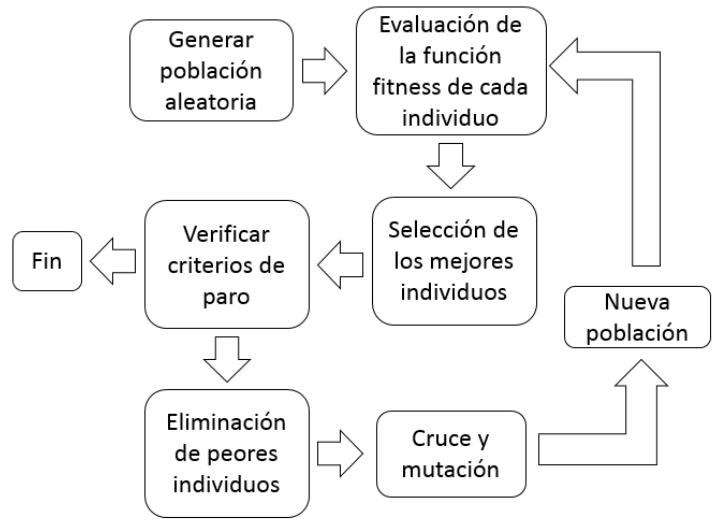

Fig. 3. Diagrama del Algoritmo genético. 
La función fitness define 4 valores que son el sobreimpulso y el tiempo de establecimiento para ambas articulaciones, en la Fig. 4 se puede ver el diagrama de la función fitness. Donde como método de integración se utilizó Euler con un paso de 0.001 segundos con 10 segundos de simulación para cada individuo. Se limitó el par máximo de $150 \mathrm{Nm}$ a $50 \mathrm{Nm}$ para la articulación 1 y de $15 \mathrm{Nm}$ a $10 \mathrm{Nm}$ para la articulación 2, con el fin de realizar una implementación como trabajo a futuro y evitar la saturación de los controladores.

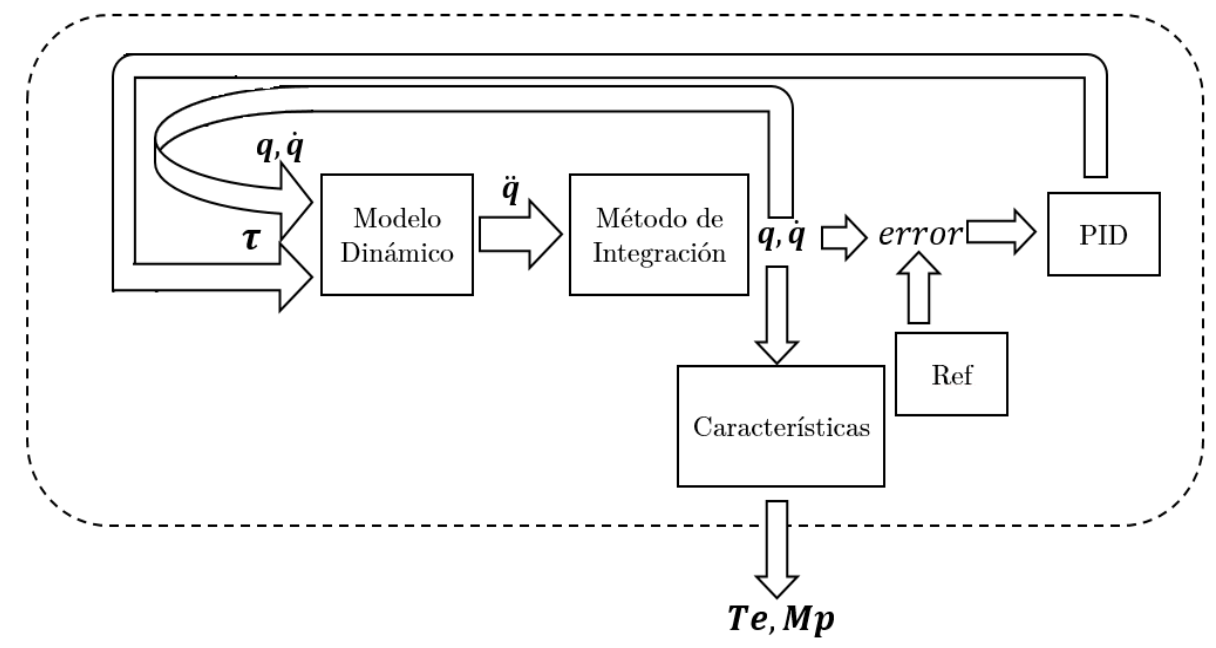

Fig. 4. Diagrama de la función fitness.

\section{Resultados}

En la Tabla 2 se muestran las ganancias encontradas. Donde se puede observar el porcentaje del sobreimpulso máximo $M_{p}$, el tiempo de establecimiento $T_{e} \mathrm{y}$ las ganancias de los controladores. De los 10 individuos finales se muestran 5 , se puede apreciar que algunas configuraciones de ganancias disminuyeron el sobreimpulso pero su tiempo de establecimiento aumento. En otros casos el tiempo de establecimiento disminuyó pero aumento el sobreimpulso. Por inspección se elige la mejor configuración de este grupo de ganancias dependiendo de los requerimientos de diseño que convengan al usuario.

Tabla 2. Ganancias encontradas.

\begin{tabular}{c|c|c|c|c|c|c|c|c|c}
\hline $\begin{array}{c}\boldsymbol{M}_{\boldsymbol{p} \mathbf{1}} \\
\boldsymbol{\%}\end{array}$ & $\begin{array}{c}\boldsymbol{T}_{\boldsymbol{e} \mathbf{1}} \\
(\mathbf{s e g})\end{array}$ & $\begin{array}{c}\boldsymbol{M}_{\boldsymbol{p} \mathbf{2}} \\
\boldsymbol{\%}\end{array}$ & $\begin{array}{c}\boldsymbol{T}_{\boldsymbol{e} \mathbf{2}} \\
(\mathbf{s e g})\end{array}$ & $\boldsymbol{K}_{\boldsymbol{p} \mathbf{1}}$ & $\boldsymbol{K}_{\boldsymbol{i 1}}$ & $\boldsymbol{K}_{\boldsymbol{d} \mathbf{1}}$ & $\boldsymbol{K}_{\boldsymbol{p} \mathbf{2}}$ & $\boldsymbol{K}_{\boldsymbol{i} \mathbf{2}}$ & $\boldsymbol{K}_{\boldsymbol{d} \mathbf{2}}$ \\
\hline 18,25 & 5.55 & $1 \mathrm{e}-6$ & 0.16 & 11.07 & 77.37 & 8.07 & 145.1 & 10.3 & 5.52 \\
$2 \mathrm{e}-8$ & 3.23 & 11.76 & 1.19 & 31.4 & 55.91 & 6.75 & 89.7 & 121 & 3.9 \\
33.32 & 9.92 & 1.88 & 0.15 & 6.59 & 83.61 & 7.78 & 135.7 & 6.31 & 4.48 \\
1.37 & 1.34 & 5.34 & 0.327 & 8.02 & 51.73 & 8.38 & 76.55 & 42.2 & 3.52 \\
11.41 & 2.32 & 1.68 & 0.46 & 97.97 & 133.05 & 31.26 & 241.83 & 31.87 & 31.87 \\
\hline
\end{tabular}


Eligiendo la siguiente configuración de ganancias por sus tiempos de establecimiento y simulando el sistema a 5 segundos con una referencia de 1 radian para ambos controladores:

$$
\begin{array}{lll}
K_{p 1}=97.97 & K_{i 1}=133.05 & K_{d 1}=31.26 \\
K_{p 2}=241.83 & K_{i 2}=31.87 & K_{d 2}=31.87
\end{array}
$$

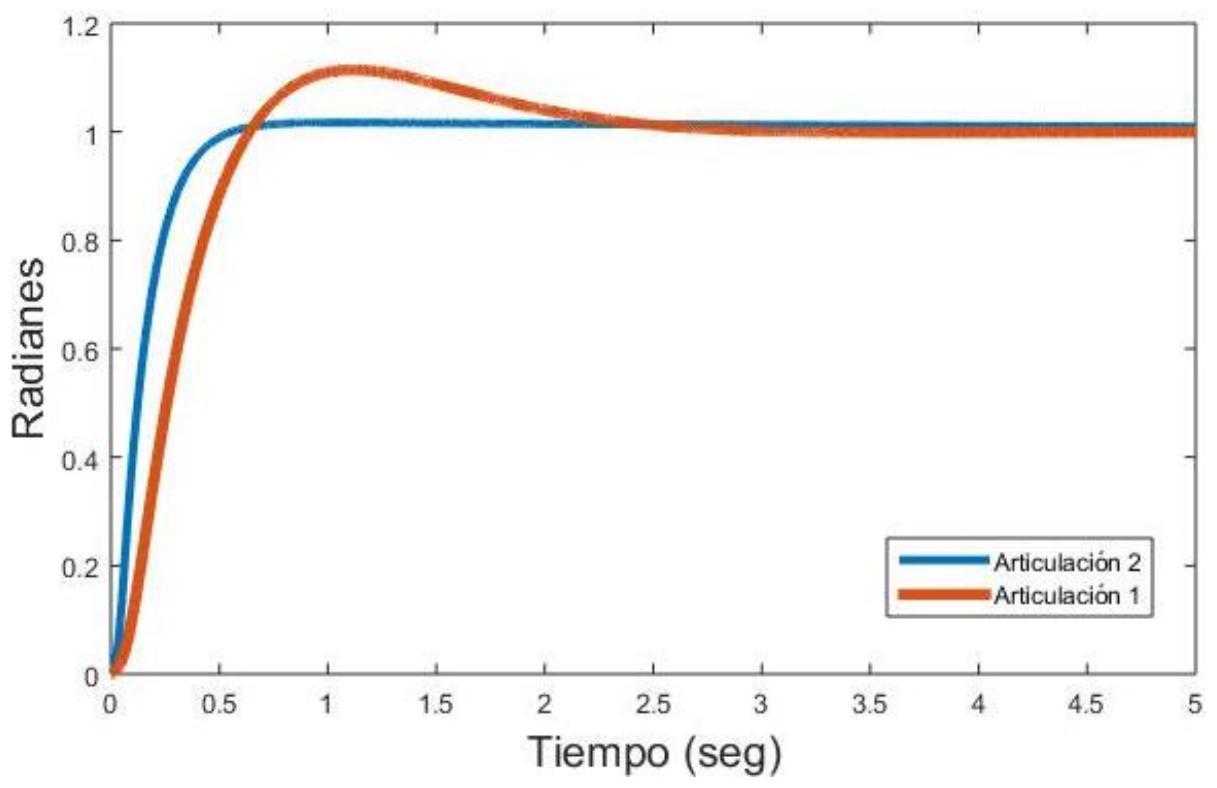

Fig. 5. Respuesta del sistema.

Se graficó la respuesta del sistema, observe que el sobreimpulso máximo (Fig. 5) no supera el $15 \%$ y el tiempo de establecimiento no rebasa 2.5 segundos para el primer eslabón. Para el segundo eslabón el sobreimpulso no superó el $2 \%$ y el tiempo de establecimiento no rebasa 0.5 segundos.

\section{Conclusiones}

El uso de los algoritmos genéticos para la optimización es viable debido a que a partir de un espacio de búsqueda grande con ciertas restricciones es posible encontrar la configuración que optimiza el desempeño de la respuesta del sistema, es decir, las ganancias que reducen el tiempo de establecimiento y el sobreimpulso máximo.

Los resultados obtenidos presentan sobreimpulsos menores al 15\%, tiempos de establecimiento menores a 2.5 segundos tomando en cuenta que es un sistema MIMO, no lineal y que no se usó el par máximo para ambas articulaciones.

La desventaja de la primera sintonización, es que requiere de un tiempo considerable (1 hora) debido a que el rango de búsqueda, tamaño de la población y número de generaciones son grandes. Una vez que se han encontrado ganancias aceptables se puede reducir el rango de búsqueda. Como trabajo a futuro se 
implementará el sistema en un Controlador digital de señales (DSC, por sus siglas en ingles) para tener una mayor aproximación a la implementación con un robot manipulador real.

Agradecimientos. David Bedolla Martínez, con número de becario 577881, agradece al CONACyT por la beca otorgada. Así mismo se agradece al programa CátedrasCONACYT con el proyecto 621 "Desarrollo de mecanismos robóticos para rehabilitación física", por la participación en éste trabajo.

\section{Referencias}

1. Santos, S.O.: Sintonización de un controlador PID basado en un algoritmo heurístico para el control de un Ball and Beam. Tesis, Universidad Autónoma de Querétaro (2014)

2. Åström, K.J., Hägglund, T.: The future of PID control. Control Engineering Practice, No 9, pp. 1163-1165 (2001)

3. PID tuning using extremum seeking: online, model free performance optimization. IEEE Control Systems, No. 79, pp. 70-79, March (2006)

4. Chang, W.D.: A multi-crossover genetic approach to multivariable PID controller tuning. Expert systems with applications, No. 32, pp. 620-626 (2007)

5. Iruthayarajan, M.W., Baskar, S.: Evolutionary Algorithms based design of multivariable PID controller, Expert Systems and applications, no 36, pp. 9159-9167 (2009)

6. Luna-Ortiz, C.: Diseño e implementación de un algoritmo genético en FPGA para sintonización de controladores PID, Tesis, Universidad Autónoma de Querétaro (2011)

7. Oha, S.-K., Janga, H.-J., Pedrycz, W.: The design of a fuzzy cascade controller for ball and beam system: A study in optimization with the use of parallel genetic algorithms. Engineering Aplications of Artificial Intelligence, No. 22, pp. 261-271 (2009)

8. Hultmann Ayala, H.V., dos Santos Coelho, L.: Tuning of PID controller based on a multiobjective genetic algorithm applied to a robotic manipulator. Expert System with Applications, No. 39, pp. 8968-8974 (2012)

9. Kelly, R., Santibáñez, V.: Control de movimiento de Robots Manipuladores. Ed. Pearson Educación (2003)

10. Leyva, F. H. R., Gaspar, L. A. P., Espinosa, F. S.: Simulación de un Robot de Dos Grados de Libertad Usando "Hardware-in-the-loop" con Base en Instrumentación Programable, Pistas educativas (2015)

11. Cortés, F. R.: Robótica, Control de Robots Manipuladores. México D.F.: Alfaomega Grupo Editor, febrero (2013) 